\title{
Overcoming selfishness: reciprocity, inhibition, and cardiac-autonomic control in the ultimatum game
}

\author{
Stefan Sütterlin ${ }^{1,2 *}$, Cornelia Herbert' ${ }^{2}$, Michael Schmitt ${ }^{3}$, Andrea Kübler $^{2}$ and Claus Vögele ${ }^{1}$ \\ 1 Integrative Research Unit on Social and Individual Development (INSIDE), University of Luxembourg, Walferdange, Luxembourg \\ 2 Department of Psychology I, University of Würzburg, Würzburg, Germany \\ 3 Fachklinik Enzensberg, Hopfen am See, Germany
}

\section{Edited by:}

Colin G. DeYoung, University of

Minnesota, USA

Reviewed by:

Alexander Weiss, The University of

Edinburgh, UK

Raymond A. Mar, York University,

Canada

*Correspondence:

Stefan Sütterlin, Integrative Research

Unit on Social and Individual

Development (INSIDE), Campus

Walferdange, Route de Diekirch, L-7220

Walferdange, Luxembourg.

e-mail:stefan.suetterlin@uni.lu
The processes underlying decision-making in response to unfair offers in the ultimatum game (UG) have recently been discussed in light of models of reciprocity and fairness-related behavior. It has been suggested that behavior following norm-oriented, internalized expectations of reciprocity requires overcoming economic self-interest. In this study we investigated both, behavioral and peripheral-physiological indicators of inhibitory capacity related to neuronal networks that are likely to be involved in the behavioral response to unfair offers. Both heartrate variability as an index of inhibitory capacity, and performance in a motor response inhibition task predicted rejection of unfair offers in an UG, suggesting an important role of inhibitory processes in overcoming economic temptations and regulating behavior conforming to social norms of reciprocity and fairness. The role of parasympathetic activity as a physiological traitmarker predicting inter-individual differences in the rejection of unfair offers is discussed.

Keywords: ultimatum game, frontal inhibition, heart-rate variability, decision-making, reciprocity

\section{INTRODUCTION}

Behavioral economics has traditionally ignored "irrational" decisionmaking as a concept as its models are mainly rooted in game theory, which conceptualizes humans as rationally acting individuals. Only recently, the importance of emotions on decision-making has begun to be acknowledged (Camerer, 2007). In addition, interdisciplinary investigations have started to combine behavioral economics and research on emotions and individual differences (Sanfey, 2007). A behavioral paradigm that triggered decades of research is the ultimatum game (UG), which has proven to be a useful tool to distinguish between rational and emotionally motivated decisions (Güth et al., 1982; Sanfey, 2007). The UG has been shown to be a reliable paradigm for the investigation of decision-making processes in social contexts, in which a conflict between economic self-interests and perceived unfairness is created. In the UG, a player (responder) receives an offer from a second player (proposer). The proposer makes an offer to split a previously granted fund, e.g., a sum of money. If the responder accepts this offer, both players are paid accordingly. If the responder rejects the proposer's offer, neither player is paid. If the money is split in an unfair way (i.e., the responder is confronted with less than 50\% of the endowment), the probability of a rejection by the responder increases, despite this being against their economic interests.

Beyond the investigation of general psychological processes in neuroeconomics, an increasing number of studies have started to investigate individual differences in personality factors affecting behavioral tendencies observed in the UG, and their psychophysiological correlates. The majority of studies on inter-individual differences in the UG has concentrated on emotional processing such as appraisal and response strategies in response to unfair offers (Kirk et al., 2011; Vögele et al., 2010). The present study aims to extend these approaches by taking the perspective of self-regulation, i.e., the regulation of the emotional impact on decision-making processes by applying behavioral and peripheral-physiological trait measures of inhibitory control. Heartrate variability (HRV) as a measure of inhibitory control (Thayer and Lane, 2009) has very recently been suggested to provide a measure of inter-individual differences that are important for the outcome in the UG, but have been inaccessible in traditional economic experiments or behavioral economic studies (Dulleck et al., 2011).

Neuroimaging studies have provided insight into the neural processes determining decision outcomes in the UG. Sanfey et al. (2003) found activation of brain regions in the prefrontal cortex, responsible for inhibitory processing, to predict acceptance or rejection of an unfair offer in the UG. The emphasis of research investigation factors that influence decision-making in the UG has mainly focused on emotional factors in reaction to the offer and the identification of brain regions processing this emotion information, such as the anterior region of the insular cortex (Sanfey et al., 2003) and the sympathetic branch of the autonomic nervous system related to emotional arousal (ANS, Wout et al., 2006). Recently, the role of prefrontal functions has come into focus. In a study by Knoch et al. (2006) the authors experimentally manipulated activation of the dorsolateral prefrontal cortex (DLPFC) using low-frequency, repetitive transcranial magnetic stimulation (rTMS). Experimentally induced lateralized disruption of these frontal cortical regions during the UG lead to a substantial reduction of the tendency to reject unfair offers. Moreover, the right DLPFC was identified to be involved in fairness-related behaviors, suggesting that prefrontal cortical regions of particular importance for inhibition are positively associated with the rejection of unfair offers in the UG. The rejection of offers runs against the responders economic interests and induces a loss aversion that needs to be overcome (Kahneman and Tversky, 1979). Results from the neuroimaging studies (Sanfey et al., 2003) outlined above suggest that rejection of an unfair offer, which violates societal norms of 
reciprocity and fairness, requires "inhibition" of economic selfinterest in terms of material gain. Knoch et al. (2006) use the term "inhibition" as a "convenient short term for top-down control (or executive control), whose overall effect is a reduction in the weight of self-interested impulses on an individual's action (p. 829)." The authors suggest that inhibitory prefrontal areas are part of a network that modulates the relative impact of fairness motives and self-interest goals on decision-making.

The question remains, however, whether basic inhibitory processes are indeed involved in overcoming selfish action tendencies in the UG and if inhibitory processes and their consequences on decision-making behavior in the UG can be investigated by measures other than brain imaging methods. In the present study we applied two established measures of inhibitory capacity: motor response inhibition and cardiac-vagal control. Both measures are considered reliable measures of inhibitory capacity (Li et al., 2006; Thayer and Lane, 2009). They are positively related to self-control and should thus, following the argumentation of Knoch and Fehr (2007), be positively associated with fairness-related behavior in the UG. Previous research on psychophysiological correlates of decision-making in the UG focused on measures of physiological arousal like electrodermal activity or blood pressure and usually described the processes accompanying the responder's decision like for instance anger (Wout et al., 2006; Ohira, 2010; Vögele et al., 2010). Instead of investigating event-related, phasic responses in the UG, we conceptualized inhibitory capacity as an individual personality trait, assessed as resting HRV independently from the UG, and hypothesized resting HRV to predict decision outcomes in terms of rejected offers.

Heart-rate variability is a reliable, non-invasive measure of prefrontal inhibitory control and self-regulation. It is mediated parasympathetically (vagally) and obtained via electrocardiographic recording during rest (Segerstrom and Solberg Nes, 2007; Thayer and Lane, 2009). Numerous research findings support the notion that HRV can be considered a trait-marker of an individual's inhibitory capacity and shows high temporal stability (Sinnreich et al., 1998; Tarkiainen et al., 2005) and a high degree of heritability (Singh et al., 2001; Wang et al., 2009). Functional neuroimaging studies have provided evidence for the neural networks modulating HRV, and these findings stress the relevance of the involvement of inhibitory prefrontal cortical areas such as the DLPFC (Napadow et al., 2008) mediating individual's resting HRV. As a physiological trait, HRV has been conceptualized as an index of not only prefrontal neural functions such as inhibitory control (Thayer and Lane, 2009), but also as an index of emotional adaptation and self-regulation (Thayer et al., 2009). This personality trait of self-regulation can be reliably assessed in experimental settings evoking behavioral control (Schmeichel and Zell, 2007) or by HRV recordings obtained under resting conditions (Segerstrom and Solberg Nes, 2007). In the present study the inhibitory parasympathetic (vagal) branch of the ANS mediating prefrontally induced oscillations of cardiac cycle times is conceptualized as important determinant of decision-making outcome in the UG, thus introducing a measure of inhibitory control which has been demonstrated to be highly relevant for the prediction of behavioral performance (Hansen et al., 2003), emotion regulation (Appelhans and Luecken, 2006; Friedman, 2007), and emotional control in decision-making (Sütterlin et al., 2011).
The central autonomic network model (CAN; Benarroch, 1993; Thayer and Lane, 2009) specifies the neurophysiological principles of cortico-cardiac interaction, i.e., prefrontal function and cardiac-autonomic control. Experimental disruption of the DLPFC via inhibitory rTMS has been shown to result in disinhibition of cardiac-autonomic control, as reflected in increased heart-rate and decreased vagal activity, i.e., reduced power in the high-frequency component of HRV (Städtgen, 2008). In the context of decisionmaking, both, activity level of the DLPFC (De Martino et al., 2006) and HRV (Sütterlin et al., 2011) have been shown to play a role in overcoming affective biases in decision-making tasks.

In summary, activation in prefrontal areas is known to be closely linked to inhibitory capacity and has been shown to be positively related to the rejection of unfair offers in the UG. These findings have been interpreted as reflecting top-down control of economic self-interest for the sake of fairness and reciprocity (Knoch and Fehr, 2007). Imaging data and experimental disruption of prefrontal activation conform to this notion (Sanfey et al., 2003; Knoch et al., 2006). In the present study, we follow this interpretation by introducing measures of inhibitory control; we predict a positive relationship between inhibitory control as trait and the number of rejected unfair offers, i.e., the successful and effortful overcoming of economic self-interest. Inhibitory trait capacity is operationalized as resting HRV and inhibitory performance in a motor response inhibition task.

By applying physiological and behavioral trait measures of inhibitory control and quantifying their association with rejection frequency in the UG, this study is the first to compare selfregulatory capacity with responses to unfair offers by introducing HRV as a peripheral-physiological index for inhibitory control in decision-making in the UG.

\section{MATERIALS AND METHODS PARTICIPANTS}

Thirty-five adults were recruited from the undergraduate student population of the University of Würzburg and outside the University via advertisements. Seven volunteers from the student sample were familiar with the UG and, therefore, were not included in the dataset. Out of the remaining 28 adults ( 18 women) 21 were undergraduate students and seven from outside the University. Participant's age ranged from 18 to 34 years $(M=23.0 ; \mathrm{SD}=3.67)$. Exclusion criteria were current and previous psychiatric, neurological, or cardiovascular diagnoses and medication affecting the central nervous or cardiovascular system. Participants received a financial compensation and gave informed consent. The local ethics committee of the University approved the study protocol, and the study was conducted in accordance with the Helsinki Declaration of 1975 (as revised in 1983).

\section{MATERIAL AND EXPERIMENTAL TASKS Motor response inhibition}

For the assessment of motor response inhibition we used the stopsignal task (SST, Logan et al., 1997). "GO”-stimuli were consisted of letters "S" or "B," which were presented centered on a 19' computer screen using E-Prime software (v2.0, Psychology Software Tools, Inc., Pittsburgh, PA, USA, 2007). “GO”-stimuli were presented for $500 \mathrm{~ms}$ and followed by an inter-trial interval (ITI) of 
$1500 \mathrm{~ms}$. The total number of trials was 600; in 150 trials (25\%) the "GO"-stimulus was followed by an acoustic stop signal $(1000 \mathrm{~Hz}$, $500 \mathrm{~ms}$ ), i.e., Stop-trials. Stimulus onset asynchrony (SOA) between GO- and STOP-signal in the stop-trials was 100, 200, or $300 \mathrm{ms,}$ respectively and varied by using a performance-related staircasetracking algorithm (Boecker et al., 2007). Correct no-responses led to an increase of SOA by $100 \mathrm{~ms}$ up to a maximum of $300 \mathrm{~ms}$, false reactions in stop-trials led to a decrease of SOA by $100 \mathrm{~ms}$ down to a minimum of $100 \mathrm{~ms}$. This was to ensure a similar level of subjective difficulty for all participants. In trials with longer delays between "GO"-stimulus and "STOP"-sound the inhibition of an already initiated motor response is more difficult than in trials where the "STOP"-sound follows immediately after the "GO"stimulus, interrupting the motor response on an earlier level. The underlying horse-race-model (Logan and Cowan, 1984) is based on the assumption of two independent processes for the response and its inhibition, making the variation of the SOA critical for the adjustment of subjective difficulty.

\section{Physiological assessment}

Electrocardiogram (ECG) was recorded using the Einthoven lead I configuration with disposable electrodes attached to the left and right wrists. Participants were instructed to relax and close their eyes while ECG monitoring for a period of $10 \mathrm{~min}$. ECG raw data were recorded using a g. USBamp amplifier (sampling rate $500 \mathrm{~Hz}$; g.tec, Graz, Austria).

\section{Experimental protocol}

In an effort to disguise the real purpose of the experiment participants were asked upon arrival at the laboratory to step in for another participant to support a research project on negotiations with students of economical sciences, just before the "actual" experiment he/she had signed up for was due to begin. The participant's role was described as the "assisting" counterpart in an experimental setting, in which the focus of the observation was on the other person, who was placed in an adjacent room. Room signs and temporary absence of the experimenter increased the credibility of the (fictitious) partner. The basic rules of the UGi.e., options and their consequences - were explained. The experimenter left the room to collect the other (fictitious) participant's offer in a closed envelope, in which the offered ratio was marked on a prepared sheet. Not transparent for the actual participant, all offers started with a fair offer of 50:50 Cent, followed by eight subsequent offers, out of which the four ratios 60:40, 70:30, 80:20, and 90:10 were each chosen twice in randomized order. The randomization procedure was intended to minimize carry-over effects of ratio-sequences and potential assumptions on causal relations and expectations by the participant. After confrontation with the offer, the responder (participant) replied by marking the corresponding answer ("REJECT" vs. "ACCEPT") underneath the offer on the prepared sheet, enclosed the sheet in the envelope with which the experimenter left the room and pretended to bring the participant's offer to the fictitious co-player. Instead of a computer program, a (fictitious) human co-player was chosen to increase the social relevance of the task and to ensure participants involvement in the task with sufficient associated emotional intensity (see Wout et al., 2006).
After completion of the UG, the physiological assessment and the SST were carried out after a recovery break of 15 min before participants were debriefed and manipulation and credibility checks were completed.

\section{Manipulation and credibility check}

Perceived anger and perceived unfairness were rated on visual analog scales for each ratio after completion of the UG to avoid systematic biases and reflections on the study's purpose during UG performance. Scales were presented with anchors "very fair"/"very unfair" and "I felt not angry at all”/“I felt very angry," respectively. Subsequently, participants were debriefed and asked whether they were familiar with the UG or had previously taken part in a similar study.

\section{Data reduction and analysis}

Stop-signal task. Go-signal reaction time, stop-signal reaction time (SSRT) and percentage of correctly inhibited reactions in STOPtrials were calculated following the recommendations made by Logan (Logan and Cowan, 1984; Logan, 1994). SSRT estimates the speed of the inhibitory process in milliseconds, with lower values reflecting faster inhibitory processing. Independent samples $t$-tests were calculated to check for sex differences in SSRT and rejection frequency.

Heart-rate variability. Offline analyses included the extraction of QRS complexes and interbeat intervals (IBI) from ECG recordings. Artifacts were detected via an individually calculated distribution-related threshold criterion (Berntson et al., 1990; Berntson and Stowell, 1998), deleted, and missing values were estimated via interpolation of neighboring IBIs. The last $5 \mathrm{~min}$ of the 10 -min recording session were chosen for HRV analysis in order to ensure that data reflected resting conditions. Artifact detection and correction was performed using the ECG processing software ARTiiFACT (Kaufmann et al., 2011). Statistical parameters of HRV (Task Force of the European Society of Cardiology, 1996; Allen et al., 2007) were calculated using the Kubios HRV Analysis 2.0 software (Niskanen et al., 2004). Time domain measures (for an overview: Task Force of the European Society of Cardiology, 1996) included mean heartrate, RMSSD (square root of the mean squared differences of successive NN intervals) and pNN50 (the proportion derived by dviding NN50 by the total number of NN intervals; NN intervals: elapsed time between subsequent ECG-R-peaks in milliseconds). Spectral frequency measures were derived using fast fourier transformation (FFT). Frequency bands were labeled as recommended by the Task Force of the European Society of Cardiology (1996) as highfrequency (HF, 0.15-0.4 Hz) and low-frequency (LF, 0.04-0.15 Hz) and expressed in power $\left(\mathrm{ms}^{2}\right)$ and normalized units (n.u.). Spectral frequency measures and time domain measures were used as indicators for cardiac-vagal tone and thus as physiological markers of inhibitory capacity. Both, time- and frequency domain measures of HRV have been shown to have high temporal stability and reproducibility (Task Force of the European Society of Cardiology, 1996).

Statistical analysis. Sex differences in the count of rejected offers (CRO), motor inhibition, and HRV were tested by independent samples $t$-test. Pearson's correlations between CRO and measures of inhibition (SSRT, HRV parameters) were calculated. Best predictors 
of CRO (pNN50, SSRT) were entered into a regression analysis. Both predictors were entered at once. In an additional regression analysis possibly confounding influences of sex and age on the regression model were investigated.

\section{RESULTS MANIPULATION CHECK}

Self-report measures of perceived unfairness and experienced anger increased with offers becoming progressively unfair, indicating a negative association between reciprocity and emotional impact (Figure A1 in Appendix). CRO increased with decreasing amount of shared money (Figure A2 in Appendix).

\section{CREDIBILITY}

Seven participants were familiar with the UG, although none of them had taken part in an UG before. All seven individuals were excluded from data analysis.

\section{INHIBITORY CAPACITY AND REJECTION RATES}

Correlation analyses between $\mathrm{CRO}$ and time domain measures of vagally mediated HRV measures of inhibitory control (RMSSD, pNN50) revealed significant positive associations of medium effect size (Figure A3 in Appendix). For frequency domain measures of the HRV correlation coefficients were only marginally significant and of moderate effect size (Table A1 in Appendix). There were no sex differences in any of the reported HRV parameters (all $p>0.30$ ) or in rejection frequency $(t=0.72, \mathrm{df}=26, p=0.48, d=0.25)$. Age was not associated with rejection frequency $(r=-0.02, n=28$, $p=0.93)$ or the inhibitory measures SSRT $(r=0.12, n=28, p=0.54)$ and the HRV indicators $(r \leq 0.25, n=28, p \geq 0.20)$.

Mean reaction time to GO-trials was $433 \mathrm{~ms}(\mathrm{SD}=51 \mathrm{~ms})$. Inhibitory control as indexed by percentage of successfully inhibited motor reactions in the SST showed the expected positive correlation with CRO (Figure A4 and Table A1 in Appendix). SSRT showed similar medium effect sizes when correlated with CRO (Table A1 in Appendix). No sex differences in SSRT were found $(t=0.845$, $\mathrm{df}=26, p=0.41)$.

The intercorrelation between both measures of inhibition (HRV, SST) was not significant and for no HRV parameter higher than $r=0.197$, $\mathrm{df}=27, p=0.17$ (for pNN50), indicating complementary roles of both measures and suitability for a multiple regression with both inhibitory measures as predictors. In a regression analysis entering both measures of inhibitory control simultaneously, the total variance explained by the model was $36.5 \%\left(R_{\text {adjusted }}^{2}=30.7 \%\right), F(2,22)=6.32, p=0.04$. SST performance (percentage of correct inhibited motor responses) was a slightly better predictor for CRO $(\beta=0.427, p<0.04)$ than pNN50-HRV $(\beta=0.352, p=0.05)$. When age and sex were entered as covariates in the regression, the effects of interest remained largely unchanged $\left[F(2,20)=3.10, p=0.04, R_{\text {adjusted }}^{2}=25.9 \%\right]$. Neither age nor sex contributed significantly to the model (sex: $\beta=0.114, p<0.55$; age: $\beta=0.045, p<0.81)$.

\section{DISCUSSION}

Previous studies have shown that the control of economic temptation and behavioral regulation in the context of conformity with norms of fairness and reciprocity in the UG requires executive functions and the ability to inhibit pre-potent responses (Sanfey et al., 2003; Knoch et al., 2006). In line with this assumption, results from the current study suggest that inhibitory capacity as measured by the responder's HRV at rest and his other performance in a motor response inhibition task both predict decision-making patterns in the UG. Inhibitory capacity is closely linked to prefrontal activation under resting conditions, as has been shown in a large body of research, and the model of neuro-visceral integration (Benarroch, 1993; Thayer and Brosschot, 2005; Thayer and Lane, 2009). HRV as a central, physiological measure of an individual's inhibitory capacity indicates inhibition-induced self-control and has been shown to predict behavior in complex cognitive and emotional situations (Appelhans and Luecken, 2006; Segerstrom and Solberg Nes, 2007; Wojniusz et al., 2011) and effects of affect-driven decision-making biases such as emotional frames (Sütterlin et al., 2011).

The current results used HRV as a peripheral-physiological measure of inhibitory control that is presumed to be mediated by frontal cortical networks. The findings are in line with previous findings using neuroimaging (Sanfey et al., 2003) or rTMS techniques (Knoch et al., 2006). These studies demonstrated that increased prefrontal activity facilitates emotional control and promotes higher rejection rates in the UG, conform with social norms and contrary to economic self-interests (Knoch et al., 2006; Knoch and Fehr, 2007). In other words, higher prefrontal activity has been associated with a tendency to reject unfair offers in the UG. As shown previously, de-activation of frontal inhibitory activity results in reduced CRO (Knoch et al., 2006). HRV at rest is modulated by prefrontal activation and is a valid marker for regulated emotional responding (e.g., Appelhans and Luecken, 2006). The positive association between measures of an individual's frontal inhibitory capacity (HRV) and CRO in the current study appears contrary to the intuitive notion that inhibitory control of emotions such as anger is necessary to overcome the emotional impact of an unfair offer, but it is in line with the hypothesized overcoming selfishness and the anticipated loss (loss aversion as psychological cost). It could thus be argued that anticipating the emotional outcome from the responder's perspective requires a weighing of economic costs (if rejecting the offer) vs. emotional costs such as feeling exploited or threatened in one's self-image (if accepting the offer). Knoch and Fehr (2007) findings on effortful self-control to overcome economic interests can be explained by the pursuit of reciprocity and social norms, but alternatively also by the responder's attempt to reduce the emotional consequences of rejection or acceptance of the offer.

In the present study, indicators of inhibitory control predicted decision-making outcomes in the UG. Further research is needed to explore the nature of the association between inhibition-linked self-control and UG outcome. It may be surprising that the target of inhibition is economic self-interest, and not aversive emotions such as anger experience to an unfair offer. Rather, the results from the present study suggest that "rational" decision-making is not equal to "economic" decision-making, but reflects "economic management of one's emotional costs" in a given situation, requiring emotion regulation processes associated with inhibitory functions. Thus, emotional costs are an outcome of the unfair offer, but they also result from the (anticipated) experience of material loss and a violation of norms of reciprocity. They, therefore, must be overcome where economic self-interest is at stake. After all, economic interests 
and overcoming selfishness can be considered psychological costs due to the resulting experience of a loss. Thus, the rejection of an unfair offer, i.e., not receiving any payment at all, is at odds with the usually dominant goal of loss aversion (Kahneman and Tversky, 1979). Loss aversion has been shown to be negatively associated with inhibitory capacity as measured by HRV at rest (Sütterlin et al., 2011). A rational individual, therefore, needs to tap into neuronal resources of frontal inhibitory capacity, which can be conceived of as a trait and quantified as HRV at rest. The current findings suggest that vagal tonic activation assessed via HRV at rest is a peripheralphysiological trait measure of self-regulation capacity, which can contribute to the prediction of behavioral outcomes in the UG. This is in line with previous literature suggesting a close association of HRV and behavioral tendencies in complex and emotionally salient decision-making scenarios.

\section{LIMITATIONS}

The present study used only two examples of inhibition paradigms. A broader range of inhibition measures (e.g., anti-saccades, inhibitory event-related potentials obtained by EEG) and experimental variations of conflicting interests such as using high vs. low monetary incentives (variation of economic incentives) would in future studies allow further conclusions regarding the role of subjective factors in the UG and the time course of inhibitory processes in decision-making. The association of inhibitory measures and rejection frequency should be less pronounced where overcoming anticipated psychological costs is required, compared to overcoming of economic interests, as postulated by previous research. Secondly, although we focused on inhibitory processes under resting conditions rather than autonomic reactivity per se, the concomitant recording of sympathetic activity (e.g., impedance cardiography) under resting conditions as well as phasic cardiac responses during the UG might contribute to a more comprehensive picture regarding autonomic balance (e.g., Vögele et al., 2010). Nevertheless, non-invasive event-related recordings of vagal activity are methodologically questionable in less standardized settings of social interaction where the emphasis is on re-creating a realistic and authentic situation. Thirdly, this study emphasized credibility and social interaction in an authentic UG environment. This naturally limits the number of possible trials per condition (variation of the degree of unfairness) and thus does not provide reliable estimates of interaction between personality traits, degree

\section{REFERENCES}

Allen, B., Chambers, A. S., and Towers, D. N. (2007). The many metrics of cardiac chronotropy: a pragmatic primer and a brief comparison of metrics. Biol. Psychol. 74, 243-262.

Appelhans, B. M., and Luecken, L. J. (2006). Heart rate variability as an index of regulated emotional responding. Rev. Gen. Psychol. 10, 229-240.

Benarroch, E. E. (1993). The central autonomic network: functional organization, dysfunction, and perspective. Mayo Clin. Proc. 68, 988-1001.

Berntson, G. G., Quigley, K. S., Jang, J. F., and Boysen, S. T. (1990). An approach to artifact identification: application to heart period data. Psychophysiology 27, 586-598.

Berntson, G. G., and Stowell, J. R. (1998). ECG artifacts and heart period variability: don't miss a beat! Psychophysiology 35, 125-132.

Boecker, M., Buecheler, M. M., Schroeter, M.L., and Gauggel, S. (2007). Prefrontal brain activation during stop-signal response inhibition: an event-related functional near-infrared spectroscopy study. Behav. Brain Res. 176, 259-266.

Camerer, C. F. (2007). Neuroeconomics: using neuroscience to make economic predictions. Econ. J. 117, C26-C42.

of unfairness, and response. We recommend a replication of this study in a computer-assisted laboratory study where the trade-off focuses less on external validity and social interaction for the sake of a higher number of performed trials and variations, allowing for the investigation of interaction effects that might shed light on inter-individual differences in the response functions depending on levels of given unfairness. Finally, this study's sample size is low and further replications of the reported findings in larger samples are recommended.

\section{CONCLUSION}

To our knowledge, this study is the first to demonstrate the effects of cardiac parasympathetic activity at rest as a physiological indicator of decision-making on responses to unfair offers. HRV and motor response inhibition are two established markers of inhibitory capacity; when combined they explained $36.5 \%$ of the variance of individual's UG rejection rate, suggesting a crucial role of individual self-regulatory capacity when responding to unfair offers. Our findings support Knoch et al. (2006), Knoch and Fehr (2007) notion of an active inhibitory process promoting a higher rejection rate. Further research is needed to clarify whether the subject of this inhibitory process is related to economic self-interest and considerations of reciprocity, or rather is led by motives evoked by anticipatory assessment of the decision's psychological consequences.

The current findings demonstrate the predictive power of physiological indicators of inhibitory capacity on UG outcomes. It further supports previous imaging findings suggesting an active inhibitory process in favor of fairness-related behavior and reciprocity.

In general terms, these findings suggest that physiological personality traits of inhibitory capacity like HRV can contribute to a better understanding of behavioral patterns in economic paradigms. This approach goes beyond the description of emotional responses triggered by external events and explains behavioral variance by objective assessment of physiological correlates of decision-making tendencies. Furthermore, future experimental approaches applying purely event-related physiological measures (e.g., phasic cardiovascular responses) to monitor processes in the UG such as arousal (i.e., emotional responsiveness to unfair offers) might benefit from additional assessment of variables with traitcharacteristics to provide a broader picture of the determinants of behavioral outcome.

De Martino, B., Kumaran, D., Seymour, B., and Dolan, R. J. (2006). Frames, biases, and rational decision-making in the human brain. Science 313 684-687.

Dulleck, U., Ristl, A., Schaffner, M., and Torgler, B. (2011). Heart rate variability, the autonomic nervous system, and neuroeconomic experiments. $J$. Neurosci. Psychol. Econ. 4, 117-124.

Friedman, B. H. (2007). An autonomic flexibility-neurovisceral integration model of anxiety and cardiac vagal tone. Biol. Psychol. 74, 185-199.

Güth,W., Schmittberger, R., and Schwarze, B. (1982). An experimental analysis of ultimatum bargaining. J. Econ. Behav. Organ. 3, 367-388.

Hansen, A. L., Johnsen, B. H., and Thayer, J. F. (2003). Vagal influence on working memory and attention. Int. J. Psychophysiol. 48, 263-274.

Kahneman, D., and Tversky, A. (1979). Prospect theory: an analysis of decision under risk. Econometrica 47, 263-291.

Kaufmann, T., Sütterlin, S., Schulz, S. M., and Vögele, C. (2011). ARTiiFACT: a tool for heart rate artifact processing and heart rate variability analysis. Behav. Res. Methods. doi: 10.3758/ s13428-011-0107-7. [Epub ahead of print]. 
Kirk, U., Downar, J., and Read Montague, P. (2011). Interoception drives increased rational decision-making in meditators playing the ultimatum game. Front. Neurosci. 5:49. doi: 10.3389/fnins.2011.00049

Knoch, D., and Fehr, E. (2007). Resisting the power of temptations - the right prefrontal cortex and self control. Ann. N. Y. Acad. Sci. 1104, 123-134.

Knoch, D., Pascual-Leone, A., Meyer, K., Treyer, V., and Fehr, E. (2006). Diminishing reciprocal fairness by disrupting the right prefrontal cortex. Science 314, 829-832.

Li, C. S., Huang, C., Constable, R. T., and Sinha, R. (2006). Imaging response inhibition in a stop-signal task: neural correlates independent of signal monitoring and post-response processing. J. Neurosci. 26, 186-192.

Logan, G. D. (1994). "On the ability to inhibit thought and action: a user's guide to the stop-signal paradigm," in Inhibitory Processes in Attention, Memory, and Language, eds D. Dagenbach and T. H. Carr (San Diego: Academic Press), 184-239.

Logan, G. D., and Cowan, W. B. (1984). On the ability to inhibit thought and action: a theory of an act of control. Psychol. Rev. 91, 295-327.

Logan, G. D., Schachar, R. J., and Tannock, R. (1997). Impulsivity and inhibitory control. Psychol. Sci. 8, 60-64.

Napadow, V., Dhond, R., Conti, G., Makris, N.,Brown,E.N., and Barbieri,R. (2008). Brain correlates of autonomic modulation: combining heart rate variability with fMRI. Neuroimage 42, 169-177.

Niskanen, J.-P., Tarvainen, M. P., Rantaaho, P.O., and Karjalainen, P.A. (2004). Software for advanced HRV analysis.
Comput. Methods Programs Biomed. 76, 73-81.

Ohira, H. (2010). The somatic marker revisited: brain and body in emotional decision making. Emot. Rev. 2, 245-249.

Sanfey, A. G. (2007). Decision neuroscience - new directions in studies of judgment and decision making. Curr. Dir. Psychol. Sci. 16, 151-155.

Sanfey, A. G., Rilling, J. K., Aronson, J. A., Nystrom, L. E., and Cohen, J. D. (2003). The neural basis of economic decision-making in the ultimatum game. Science 300, 1755-1758.

Schmeichel, B. J., and Zell, A. (2007). Trait self-control predicts performance on behavioral tests of self-control. J. Pers. 75, 743-755.

Segerstrom, S. C., and Solberg Nes, L. (2007). Heart rate variability reflects self-regulatory strength, effort, and fatigue. Psychol. Sci. 18, 275-281.

Singh, J. P., Larson, M. G., O’Donnell, C. J., and Levy, D. (2001). Genetic factors contribute to the variance in frequency domain measures of heart rate variability. Auton. Neurosci. 90, 122-126.

Sinnreich, R., Kark, J. D., Friedlander, Y., Sapoznikov, D., and Luria, M. H. (1998). Five minute recordings of heart rate variability for population studies: repeatability and age-sex characteristics. Heart 80, 156-162.

Städtgen, M. (2008). Effects of Transcranial Magnetic Stimulation over the Dorsolateral Prefrontal Cortex on Cognitive Controland on the Autonomic Nervous System. Doctoral dissertation, RWTH, Aachen.

Sütterlin, S., Herbert, C., Schmitt, M., Kübler, A., and Vögele, C. (2011). Frames, decisions, and cardiac-autonomic control. Soc. Neurosci. 6, 169-177.

Tarkiainen, T. H., Timonen, K. L., Tiittanen, P., Hartikainen, J. E. K., Pekkanen, J., Hoek, G., Ibald-Mulli, A., and Vanninen, E. J. (2005). Stability over time of short-term heart rate variability. Clin. Auton. Res. 15, 394-399.

Task Force of the European Society of Cardiology, and The North American Society of Pacing, and Electrophysiology. (1996). Heart rate variability - standards of measurement, physiological interpretation, and clinical use. Eur. Heart J. 17, 354-381.

Thayer, J. F., and Brosschot, J. F. (2005) Psychosomatics and psychopathology: looking up and down from the brain. Psychoneuroendocrinology 30, 1050-1058.

Thayer, J. F., and Lane, R. D. (2009) Claude Bernard and the heart-brain connection: further elaboration of a model of neurovisceral integration. Neurosci. Biobehav. Rev. 31, 81-88.

Thayer, J. F., Hansen, A. L., Saus-Rose, E. and Johnsen, B. H. (2009). Heart rate variability, prefrontal neural function, and cognitive performance: the neurovisceral integration perspective on self-regulation, adaptation, and health. Ann. Behav. Med. 37, 141-153.

Vögele, C., Sorg, S., Studtmann, M., and Weber, H. (2010). Cardiac autonomic regulation and anger coping in adolescents. Biol. Psychol. 85, 465-471.

Wang, X., Ding, X., Su, S., Li, Z., Riese H., Thayer, J. F., Treiber, F., and Snieder, H. (2009). Genetic influences on heart rate variability at rest and during stress. Psychophysiology 46, 458-465.
Wojniusz, S., Vögele, C., Ropstad, E., Evans, N., Robinson, J., Sütterlin, S., Erhard, H. W., Solbakk, A. K., Endestad, T., Olberg, D. E., and Haraldsen, I. R. (2011). Prepubertal gonadotropin-releasing hormone analog leads to exaggerated behavioral and emotional sex differences in sheep. Horm. Behav. 59, 22-27.

Wout, M. V., Kahn, R. S., Sanfey, A. G., and Aleman, A. (2006). Affective state and decision-making in the ultimatum game. Exp. Brain Res. 169, 564-568.

Conflict of Interest Statement: The authors declare that the research was conducted in the absence of any commercial or financial relationships that could be construed as a potential conflict of interest.

Received: 15 April 2011; accepted: 11 July 2011; published online: 27 July 2011.

Citation: Sütterlin S, Herbert C, Schmitt M, Kübler A and Vögele C (2011) Overcoming selfishness: reciprocity, inhibition, and cardiac-autonomic control in the ultimatum game. Front. Psychology 2:173. doi: 10.3389/fpsyg.2011.00173

This article was submitted to Frontiers in Personality Science and Individual Differences, a specialty of Frontiers in Psychology.

Copyright (c) 2011 Sütterlin, Herbert, Schmitt, Kübler and Vögele. This is an open-access article subject to a nonexclusive license between the authors and Frontiers Media SA, which permits use, distribution and reproduction in other forums, provided the original authors and source are credited and other Frontiers conditions are complied with. 


\section{APPENDIX}

Table A1 |Time and frequency domain measures of HRV, stop-signal performance and their Pearson's product-moment correlations with count of rejected offers.

$\begin{array}{llllll}\text { Mean } & \text { SD } & \boldsymbol{r} & \boldsymbol{p} & \mathrm{Cl}\end{array}$

\begin{tabular}{lccccc}
\hline HRV & & & & & \\
RMSSD & 44.84 & 13.92 & $0.426^{*}$ & $<0.05$ & $0.09-0.81$ \\
pNN50 & 24.88 & 14.00 & $0.436^{*}$ & $<0.05$ & $0.09-0.81$ \\
HF $\left(\mathrm{ms}^{2}\right)$ & 729 & 471 & 0.294 & 0.073 & $0.00-0.97$ \\
HF (n.u.) & 35.01 & 17.61 & 0.282 & 0.077 & $0.09-0.67$ \\
STOP-SIGNALTASK & & & & & \\
Correct inhibitions (\%) & 71.7 & 15.6 & $0.496^{* *}$ & $<0.01$ & $0.16-0.83$ \\
SSRT & 166.9 & 33.4 & $-0.485^{* *}$ & $<0.01$ & -0.78 to -0.07
\end{tabular}

$\left({ }^{*} p<0.05:{ }^{*} p<0.01\right)$ Measures of HRV as defined by Task Force of the European Society of Cardiology (1996) represent indicators of vagal activity. Abbreviations: $S D$, standard deviation; $r$, Pearson's correlation coefficient; $p$, significance level; RMSSD, square root of the mean squared differences of successive NN intervals; PNN50, proportion derived by dividing number of intervals differing more than $50 \mathrm{~ms}$ from preceding interval by the total number of interbeat intervals; HF $\left(m s^{2}\right)$, high-frequency component (spectral power); HF (n.u.), normalized units of HF; SSRT, stop-signal reaction time; $\mathrm{Cl}$, confidence interval (95\%).

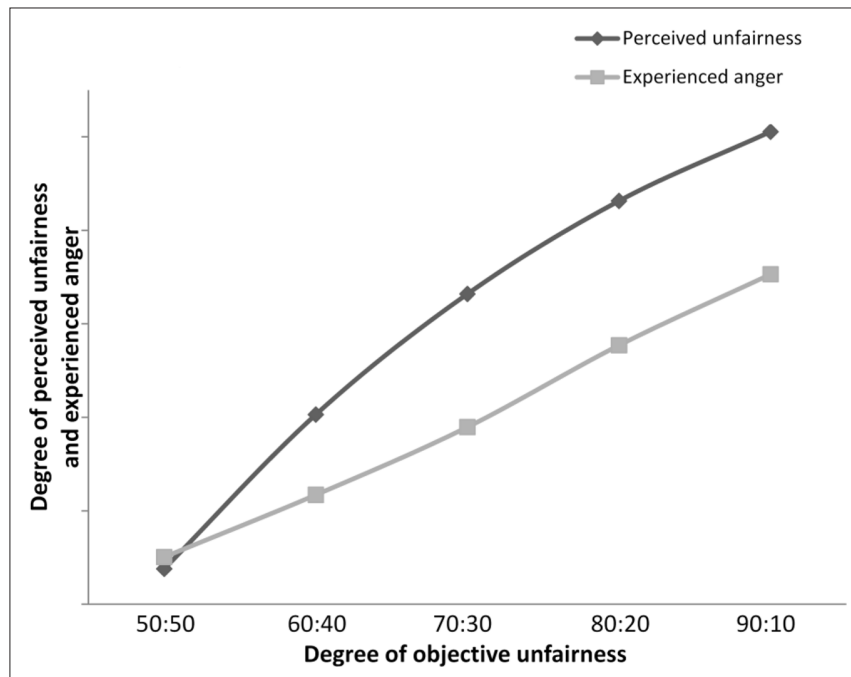

FIGURE A1 | Perceived unfairness and experienced anger. Increasing objective unfairness leads to increased experienced anger and perceived unfairness in responding participants. Vertical axis resembles values of a self-report visual analog scale with the anchors "very fair"/"very unfair" and "I felt not angry at all"/"I felt very angry."

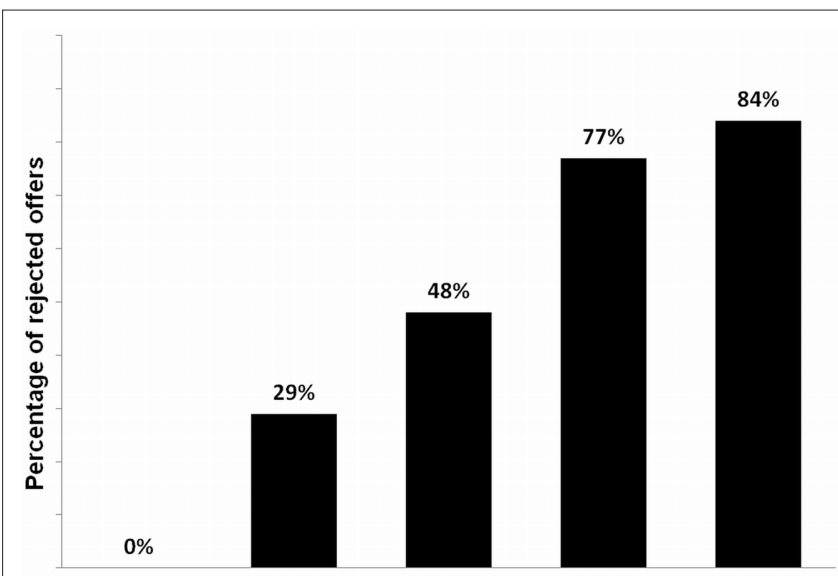

Degree of objective unfairness

FIGURE A2 | Percentage of rejected offers. The probability of a rejection of an unfair offer increases with increasing objective unfairness.

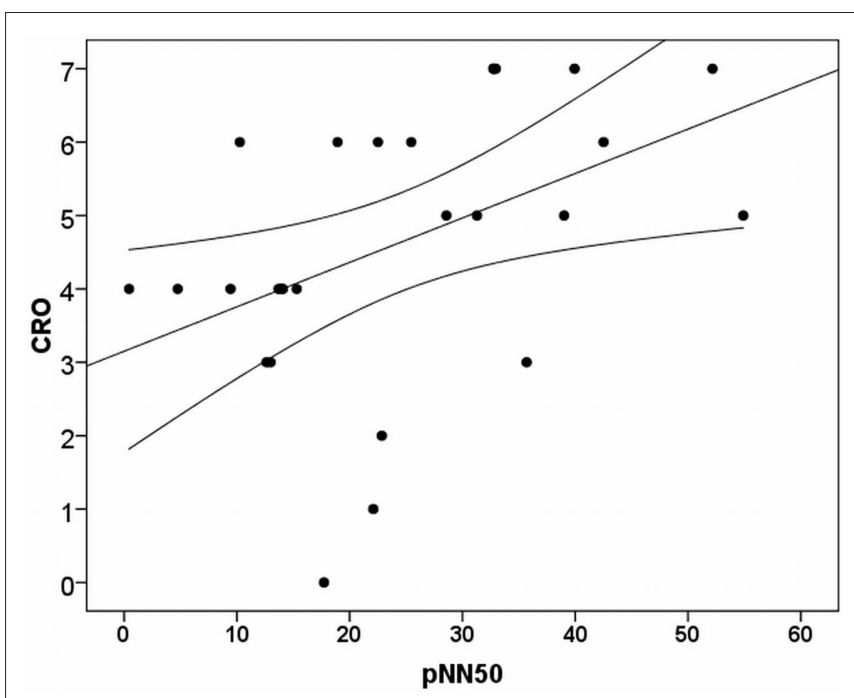

FIGURE A3 | Scatter-plot of correlation coefficients between HRV measure pNN50 and the count of rejected offers. As higher the participant's HRV at rest condition, as more likely a rejection of an unfair offer is pNN50 = proportion derived by dividing number of intervals differing more than $50 \mathrm{~ms}$ from preceding interval by the total number of interbeat intervals $\mathrm{CRO}$, count of rejected offers 


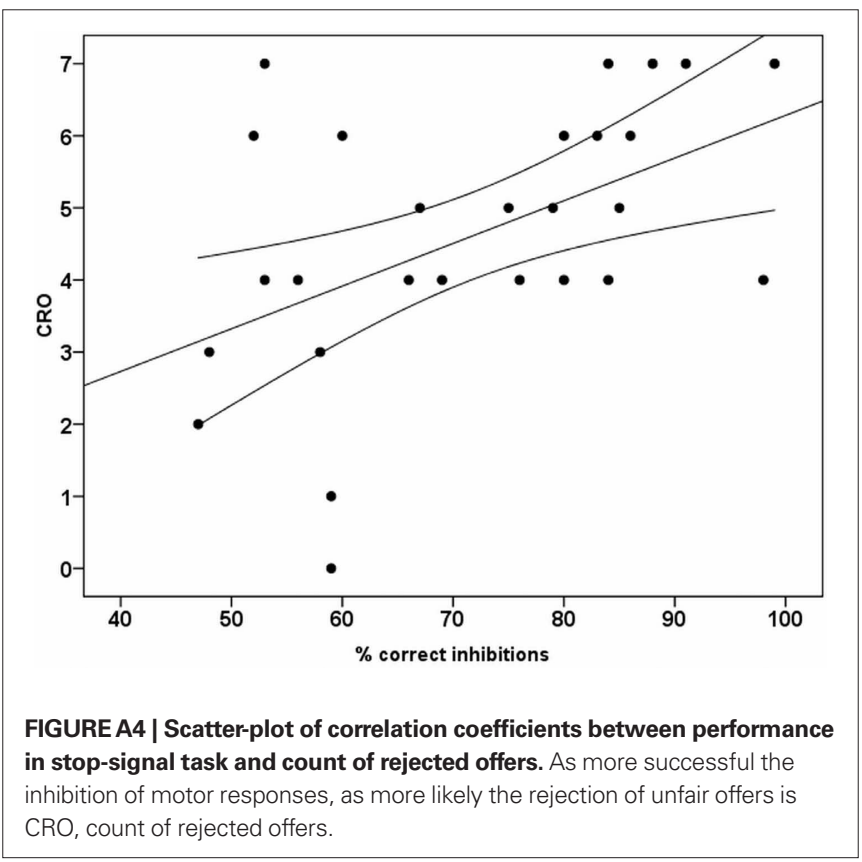

